\title{
REVIEW
}

\section{Bench-to-bedside review: Contrast enhanced ultrasonography - a promising technique to assess renal perfusion in the ICU}

Antoine Schneider1,2, Lynne Johnson ${ }^{3}$, Mark Goodwin ${ }^{3}$, Anthony Schelleman ${ }^{3}$ and Rinaldo Bellomo*1,2

\begin{abstract}
Acute kidney injury (AKI) is common in critically ill patients and associated with important morbidity and mortality. Although alterations in renal perfusion are thought to play a causative role in the pathogenesis of AKI, there is, to date, no reliable technique that allows the assessment of renal perfusion that is applicable in the ICU. Contrast-enhanced ultrasound (CEUS) is an ultrasound imaging technique that makes use of microbubble-based contrast agents. These microbubbles, when injected into the bloodstream, allow visualization of vascular structures and, with contrast-specific imaging modes, detection of blood flow at the capillary level. Some recent CEUS-derived approaches allow quantification of blood flow in several organs, including the kidney. Current generation ultrasound contrast agents have strong stability and safety profiles. Along with postmarketing surveillance, numerous studies report safe administration of these agents, including in critically ill patients. This review presents information on the physical principles underlying CEUS, the methods allowing blood flow quantification and the potential applications of CEUS in critical care nephrology, currently as a research tool but perhaps in the future as a way of monitoring renal perfusion.
\end{abstract}

\section{Introduction}

Acute kidney injury (AKI) is common and severe in critically ill patients, with a reported incidence between 1 and $30 \%$ and mortality between 28 and $90 \%$, depending

*Correspondence: rinaldo.bellomo@austin.org.au

IIntensive Care Unit, Austin Hospital, 145 Studley Road, 3084 Heidelberg, Victoria, Australia

Full list of author information is available at the end of the article on the definition used. Uchino and colleagues [1] reported an incidence of $5.7 \%$ and a mortality of $60.3 \%$ in a recent multicenter study involving nearly 30,000 critically ill patients. In patients with multiple organ failure, AKI is an independent predictor of mortality $[2,3]$.

The pathophysiology of AKI remains poorly understood. However, as renal function is intimately linked to organ blood flow, alterations in renal perfusion are considered key to the pathophysiology of AKI. Little, however, is known about renal perfusion in critically ill patients [4] or about its association with AKI. This is because methods currently available to assess and monitor renal perfusion are either inaccurate or not rapidly applicable in routine ICU patients. The physiological gold standard to estimate renal plasma flow is the calculation of para-amino-hippurate clearance. Unfortunately, this technique is inaccurate in the presence of oliguria $[4,5]$. Doppler ultrasound studies have been demonstrated to be inaccurate in estimating renal blood flow [6] and only provide information about flow in main arteries. In addition, all methods only give information about global organ perfusion and not about the renal microcirculation or on the intra-renal distribution of blood flow.

Imaging methods such as scintigraphy or magnetic resonance imaging (MRI) are much more accurate and can provide valuable information on kidney perfusion $[7,8]$. However, their use in the ICU is limited by equipment availability, costs and their requirement for extensive and prolonged patient manipulation, which is associated with risk and major logistic challenges. These techniques can be used in research protocols but are not suitable for routine use in most ICU patients and cannot be repeated several times within the same day.

A method allowing reproducible renal perfusion quantification that would be applicable at the bedside, and would be minimally invasive or even non-invasive, would be ideal in the ICU. Such a method might increase our knowledge of the correlation between renal perfusion alterations and AKI. It could also potentially help clinicians detect patients at risk of renal failure and adapt treatment early to prevent AKI. 


\section{Contrast-enhanced ultrasound Ultrasonography}

Ultrasonography is an ultrasound-based diagnostic imaging technique. It relies on the property of sound waves to reflect at interfaces of media of different densities as they travel through them - the greater the difference in density (acoustic impedance), the more echogenic the interface $[9,10]$.

Modern ultrasound equipment is portable, reasonably cheap and allows bedside examination without requiring patient manipulation. Standard ultrasound examinations are already performed in the ICU for diagnostic reasons (abdominal or liver studies, cardiac echography) and to guide interventions (central venous line placement, pleural effusion drainage). Such equipment is becoming widely available and echography is now a standard technique within most modern ICUs [11].

To evaluate circulation and blood flow, Doppler studies can be performed with standard echography equipment. The clinical use of Doppler studies is, however, limited by the lower limit of detection, its inability to detect slow flow velocity and its limited accuracy in quantifying renal blood flow. Microbubble-based contrast agents have lowered the detection threshold and now allow detection of blood in vessels as small as capillaries. Together with appropriate imaging modes and modern software, contrast-enhanced ultrasonography (CEUS) allows organ blood flow quantification.

\section{Microbubble-based contrast agents}

Gases are ideal contrast agents for echography since they are highly compressible (17,000 times more than water) and since their density is 1,000 times less than the density of blood [12]. Embedded within a shell, gases can be made to form microbubbles, which are extremely potent ultrasound reflectors, nine orders of magnitude higher than a solid particle of the same size. These microbubbles change shape when they interact with ultrasound waves, contracting during the compression (high pressure) phase and expanding during the rarefaction (low pressure) phase. At low-intermediate acoustic pressure, these microbubble oscillations result in the generation of nonlinear signals [13].

The first reported echographic contrast agent was based on air microbubbles created by agitating saline, which was then rapidly injected intravenously. It was used to examine the right heart and detect right-to-left shunts. The clinical use of agitated saline is, however, very limited due to the very short life (a few seconds) of air microbubbles and their inability to traverse the pulmonary circulation. Safety concerns were also recently raised, cerebral ischemic events being reported after use of agitated saline, probably related to the broad and uncontrolled size distribution of such bubbles [14].

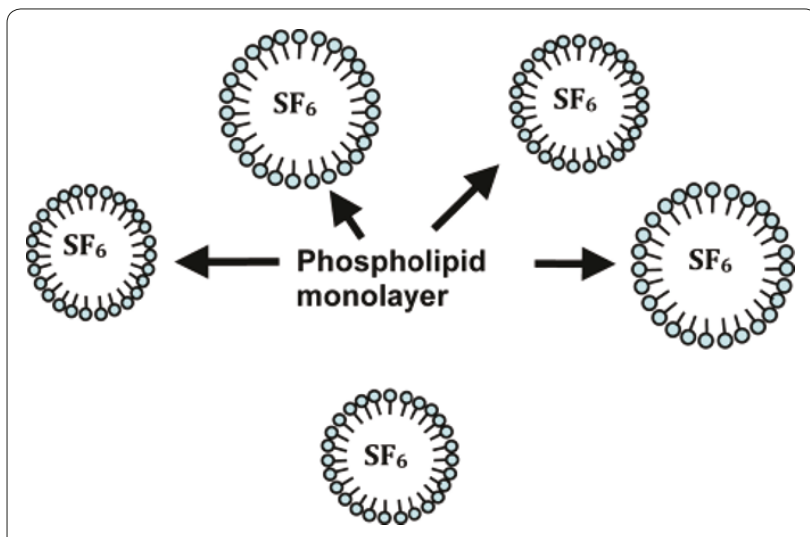

Figure 1. Schematic illustration of a microbubble contrast agent: Sonovue (courtesy of Bracco SpA).

Technological improvements have resulted in the development of small-sized shell-stabilized microbubbles with adequate half-life and the capacity to cross the pulmonary circulation (Figures 1 and 2).

The first commercially available ultrasound contrast agent was Albunex ${ }^{\circ}$ (Molecular Biosystems, Inc., USA). It was obtained by sonicating a $5 \%$ human albumin solution in the presence of air, resulting in the formation of air microbubbles stabilized by a thin denaturated albumin shell [15].

Current (second) generation microbubble contrast agents make use of inert, poorly soluble perfluorinated gases and are stabilized by phospholipids or albumin [16]. Their half-life in the circulation is a few minutes and the perfluorinated gas present in the microbubbles is totally excreted by the lungs [17].

After intravenous injection, microbubbles behave as pure blood agents as their size (1 to $6 \mu \mathrm{m}$ ) prevents them from diffusing through the endothelium. They allow vascular bed opacification and have opened the way to detecting the microcirculation [18]. The different commercially available ultrasound contrast agents and their compositions are detailed in Table 1.

\section{Optimal ultrasound equipment settings}

Parallel to the development of efficient contrast agents, novel imaging modes have been introduced. Harmonic B mode, phase or pulse inversion, power modulation, power pulse inversion, and coherent pulse sequencing are some of the contrast-specific imaging modes (also designated as 'nonlinear' imaging modes) available in modern ultrasound equipment [19]. Based on the non-linear properties of microbubbles submitted to acoustic pressure, these imaging modes use a combination of changes in pulse phase and amplitude to selectively minimize tissue echoes and enhance ultrasound contrast agent echoes. They all make use of low mechanical index (MI) imaging 


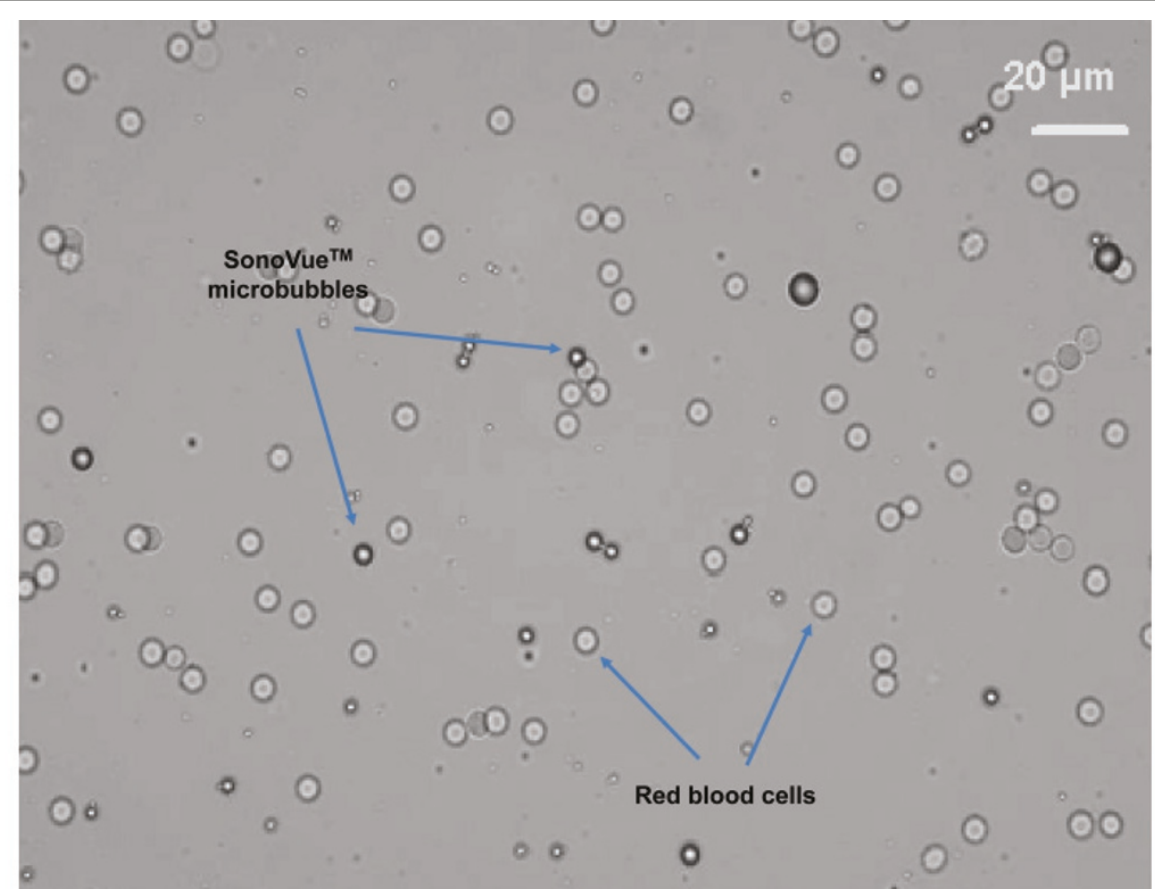

Figure 2. Optical microscopic view of microbubbles in rabbit blood (courtesy of Bracco SpA).

Table 1. Commercially available ultrasound contrast agents

\begin{tabular}{|c|c|c|c|}
\hline $\begin{array}{l}\text { Contrast } \\
\text { agent }\end{array}$ & Shell & Gas & Registered in \\
\hline Optison & Human albumin & Perfluoropropane & USA, Canada \\
\hline $\begin{array}{l}\text { Definity } \\
\text { (Luminity) }\end{array}$ & Phospholipids & Perfluoropropane & $\begin{array}{l}\text { USA, Europe, } \\
\text { Canada }\end{array}$ \\
\hline Sonovue & Phospholipids & Sulfur hexafluoride & $\begin{array}{l}\text { Canada, Europe, } \\
\text { China, India, } \\
\text { Korea }\end{array}$ \\
\hline Sonazoid & Phospholipids & Perfluorobutane & Japan \\
\hline
\end{tabular}

or intermittent imaging or both, since microbubbles can be destroyed when subjected to high acoustic pressures (for example, MI $>0.7$ ). This unique property is used for perfusion quantification in the so-called destruction (flash) refilling approach.

Today, contrast-specific modes are available on most mid- to high-end ultrasound equipment.

\section{Safety}

As for any other drug, ultrasound contrast agents have been submitted to extensive clinical investigations both for safety and efficacy before approval by national health authorities.

Since microbubbles of gas are injected into the circulation, legitimate concerns about tissue embolism can be raised, especially because, as discussed earlier, initial attempts to generate contrast by agitated saline may have been associated with embolic events [14]. However, the microbubbles in commercial ultrasound contrast agents have a much smaller and uniform size than those produced by agitating saline. They are also much more stable and do not coalesce. Therefore, they have a very low potential for embolization. This has been confirmed by intravital microscopy in the cremaster or spinotrapezius muscle with different contrast agents [21-23]. These studies show that the microvascular rheology of ultrasound contrast agents is similar to that of red blood cells and that microbubble entrapment within the capillaries is negligible and transient.

Initial post-marketing surveillance over 5 years and in more than 1 million patients has demonstrated no medically significant risk other than allergic events, which appear to occur at a rate of approximately 1 per 10,000 [20]. Central nervous system reactions have also been rarely reported and may or may not be associated with hypersensitivity reactions. Reported adverse events are generally infrequent and mild and may include headache, fatigue, palpitations, nausea, dizziness, dry mouth, altered sense of smell or taste, dyspnea, urticaria, pruritus, back pain, chest pain or rash.

In October 2007, following four deaths in patients with severe underlying conditions 1 to 12 hours after injection of Definity', a 'black box warning' stating that use of these agents was contraindicated in unstable patients was released by the US Food and Drug Administration. This initiated an intense controversy. Many clinicians still 
remained convinced that ultrasound contrast agents were safe.

Since then, many studies, including one in a very large number of patients, have been published establishing CEUS as a safe procedure. For example, Kusnetzky [24] demonstrated that the background mortality of patients undergoing contrast echography was not significantly higher than that of patients undergoing non-contrast echocardiography. Dolan and colleagues [25] studied 42,408 patients from three centers that had received contrast agents for either resting or stress echocardiography and did not find any difference in mortality or adverse events compared to matched controls. A multicenter registry including 58,254 hospitalized patients that underwent echocardiography published by Main and colleagues [26] actually showed a decrease in acute mortality compared with patients not receiving contrast agent. Wei and colleagues [27] reported a rate of severe reactions of $0.01 \%$ and no death in 78,383 patients, including 10,000 acutely ill patients (either in the ICU or with acute chest pain of possible cardiac origin) who had received ultrasound contrast agents.

Today, many investigators believe that ultrasound contrast agents can be considered safe even in unstable patients, even though the Food and Drug Administration has not yet withdrawn the black box warning. As for any drug or contrast agent, the risk of anaphylactic reaction remains present and the use of these products in unstable patients should be restricted to centers with full resuscitation capacities.

As discussed in detail in the next paragraph, blood flow quantification requires use of high MI ultrasound for very short periods of time (flashes). Some concerns have been raised about the safety of this procedure. Jiménez and colleagues [28] showed in a porcine model that repeated insonification of the kidney at high MI did not produce any histological change either immediately after the procedure or 4 hours later. There were no signs of inflammatory response and no signs of extravasation of erythrocytes from the capillary system.

\section{Blood flow quantification by CEUS}

Since microbubbles remain confined to the intravascular space, and have a rheology similar to that of red blood cells, contrast uptake as a function of time can be used to estimate quantitative perfusion parameters, such as regional blood volume or blood flow.

\section{Theories and methods}

Perfusion quantification by CEUS may be performed with a microbubble-destruction technique, introduced in 1998 by Wei and colleagues $[29,30]$. This technique was validated in a canine model with intermittent imaging with destructive frames at increasing imaging frame rates, microbubbles being continuously infused by means of a syringe pump. This allowed investigators to build a curve representing replenishment kinetics from a series of clips at different frame rates. Fitting of this curve allows derivation of two relative parameters representing perfusion in the tissue: the regional blood volume (plateau value) and blood velocity (initial slope of the replenishment curve).

The local blood flow, $F$, is thus the product of microbubble velocity by regional blood volume: $\mathrm{F} \propto \mathrm{A} \times \beta$, where A corresponds to the plateau signal intensity and $\beta$ is the initial slope of the replenishment curve. Although these data were derived from the myocardium, the authors stated that this method was applicable to any tissue accessible to ultrasound.

This technique was later extended to non-destructive imaging at low MI with the application of a few destructive frames at higher MI to completely destroy the microbubbles in the scan plane [31]. The reperfusion (or replenishment) of microbubbles in the scan plane at low MI is recorded as a single clip, and analyzed using the model of Wei and colleagues to derive perfusion parameters.

Another formal approach to the estimation of flow, developed by Arditi and colleagues [32] for the low MI imaging approach, allows improved perfusion estimates by considering an echo power signal and taking into account the ultrasound beam geometry. This approach was recently implemented in the form of a prototype software (Bracco Research SpA, Geneva, Switzerland) for off-line processing of refilling sequences. Using this software, video data are first linearized to compute an echo-power signal whose amplitude is proportional to the local contrast agent concentration. As described in the approach by Tiemann and colleagues [31], fitting of these signals after destruction allows perfusion quantification. Here, the perfusion parameters considered are: relative blood volume (rBV), mean transit time (mTT), and blood flow (rBV/mTT) (Figure 3). The software further allows the generation of parametric maps, showing the spatial distribution of perfusion parameters at the pixel level. This approach establishes a basis for extracting information on the perfusion of vascular beds in vivo, and allows relative quantification between selected areas of interest, provided that appropriate instrument calibration is implemented for the data linearization phase.

\section{Clinical applications}

Several authors have subsequently used these methods and a general good agreement has been reported in several organs and tissues.

For example, Rim and colleagues [33] were able to quantify cerebral blood flow in dogs at baseline and after cerebral blood flow alteration through hypo- or hypercapnia. A good correlation was found between $A \times \beta$ 


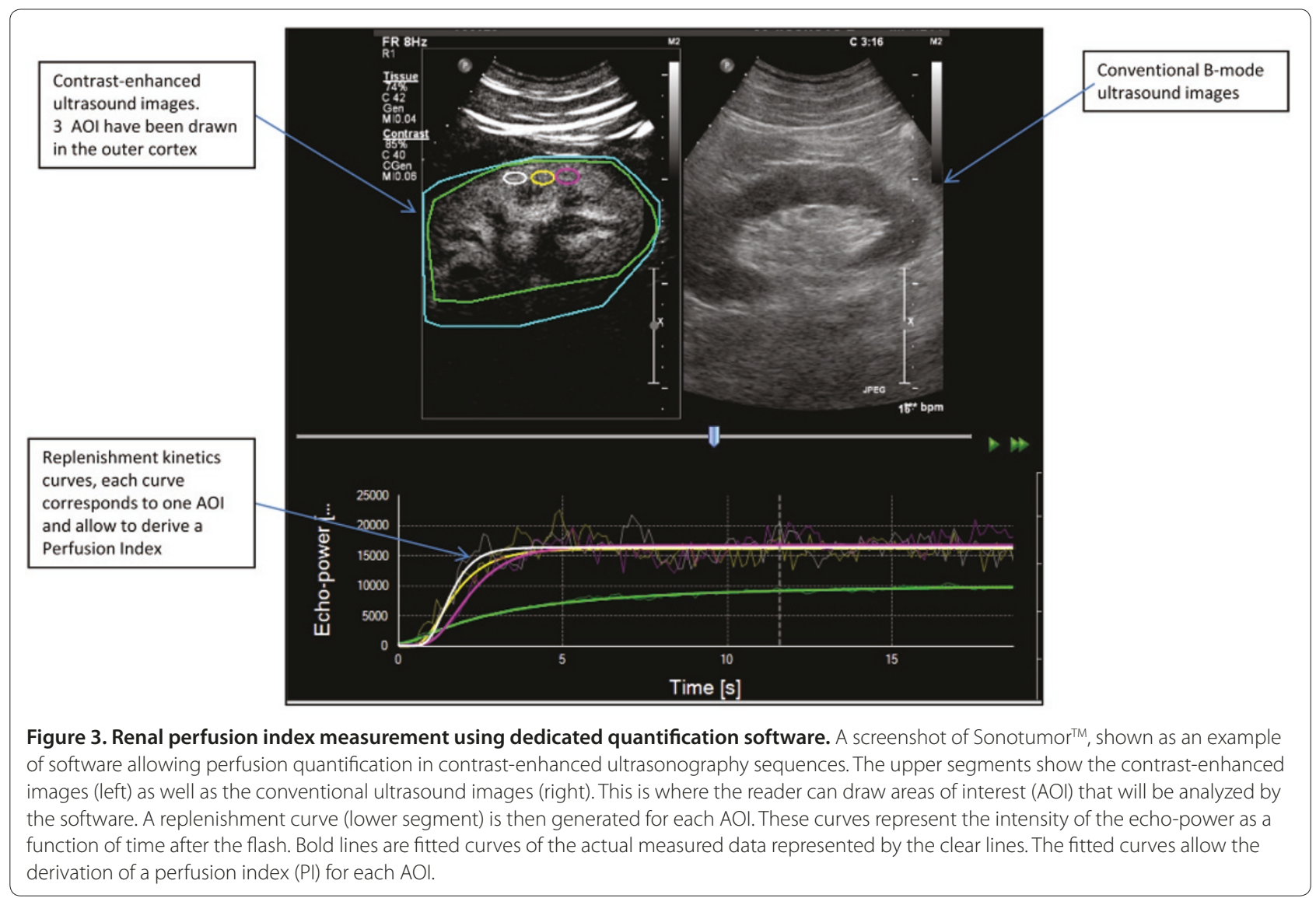

derived parameters and cerebral blood flow as measured by an accepted reference method (radiolabeled microspheres).

Vogel and colleagues [34] were able to quantify myocardial perfusion in humans. In their study, a linear correlation was found between myocardial CEUSderived parameters and PET perfusion data in healthy volunteers at rest $(\mathrm{N}=15)$ and during adenosine-induced hyperemia $(\mathrm{N}=5)$. These investigators also compared CEUS with intracoronary Doppler measurements before and during intracoronary adenosine injection in patients undergoing coronary angiograms and found good agreement with coronary flow velocity reserve.

As far as renal perfusion is concerned, Kishimoto and colleagues [35] found a congruent modification of microcirculation with an infusion of dopamine in nine healthy subjects. They subsequently used the same technique [36] to study the effect of valsartan on renal perfusion in seven healthy volunteers and found a significant increase in microbubble velocity after oral administration of valsartan that correlated well with the increase in total renal blood flow determined by para-amino-hippurate clearance $(P<0.05)$. In a recently published study, Kalantarinia and colleagues [37] tested the utility of CEUS to monitor the expected increase in renal blood flow after a high protein meal in healthy adults. They found a statistically significant increase (by $42.8 \%$ ) in renal blood flow $(\mathrm{A} \times \beta$ parameter $)$ compared with baseline $(P=0.002)$.

We recently performed a study in ten healthy volunteers evaluating changes in the perfusion index (PI; a variable that is proportional to blood flow) seen during intravenous infusion of angiotensin II and after oral captopril. We found a statistically significant and dosedependant decrease in PI during increasing doses of angiotensin II compared to baseline. The decreases in PI were already detectable when the renal plasma flow (as estimated by para-amino-hippurate clearance) decreased by $15 \%$ [38].

To further illustrate the feasibility of CEUS in the ICU, we present the example of a 66-year-old man recently studied in our center. This patient had a past medical history of non-insulin-dependent diabetes mellitus, hypertension and hypercholesterolemia. He was complaining of chest pain and an angiogram revealed a severe triple vessel disease. He was scheduled for an elective coronary artery bypass surgery. We performed a renal CEUS before and after the operation. The contrast agent injections were perfectly tolerated. The images could be acquired in less than 15 minutes, including the 


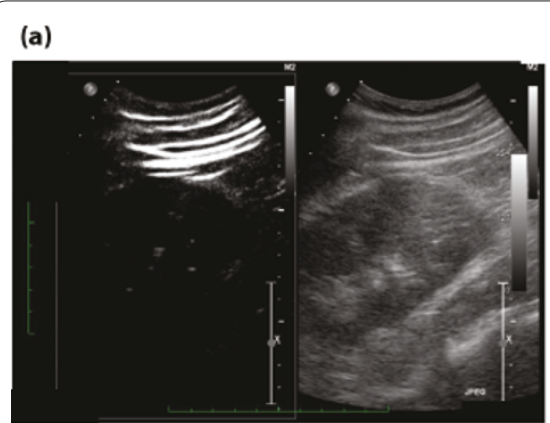

(b)

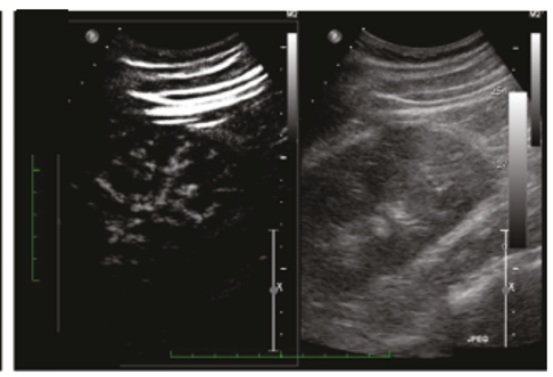

(c)

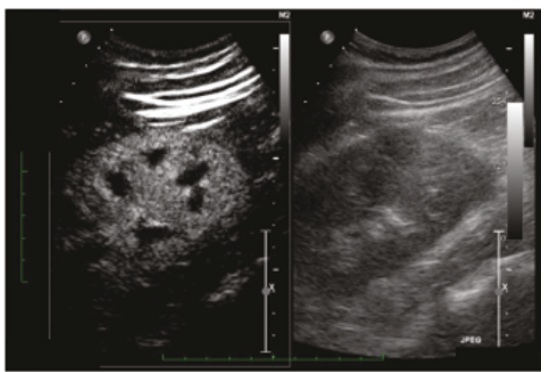

Figure 4. Example of destruction refilling sequences obtained in a 60-year-old male patient 1 hour after coronary artery bypass surgery. Each panel of the figure is divided in two; the left side shows contrast specific images and the right side standard B-mode images. (a) After the destruction flash (left), no signal is detectable in the contrast-specific image (that is, all the microbubbles have been destroyed). (b) Five seconds after destruction (left), partial replenishment of the main arteries with contrast can be noticed. (c) Ten seconds post-destruction (left), the kidney is fully replenished with contrast. No significant changes are observed in B-mode images (a-c, right).

post-operative study, which was performed within 1 hour of admission to the ICU. Figure 4 presents some still images illustrating the destruction-refilling sequences in that patient.

Interestingly, we noted a decrease in the perfusion indices from a baseline of 10,523 (before the surgery) to 7,786 (-26.0\%; Table 2). Clinically, a transient period of oliguria was noted around 12 hours post-surgery and the plasma creatinine concentration increased from 79 to $155 \mu \mathrm{mol} / \mathrm{l}$ (RIFLE F). With adequate fluid resuscitation and furosemide administration, the urinary output finally normalized but the plasma creatinine concentration remained elevated on hospital discharge $(121 \mu \mathrm{mol} / \mathrm{l})$.

\section{Experience in transplant medicine}

In renal transplant medicine, a detailed evaluation of blood flow in the subcapsular capillaries is highly desirable since the latter are primarily involved in acute rejection. Fischer and colleagues [39] examined 32 patients 5 to 7 days after kidney transplantation and were able to show that a temporal difference in the contrast agent arrival slopes between two main territories allowed the differentiation of acute graft rejection from a normal clinical course (where the slopes were uniform).

In 26 transplant patients, Schwenger and colleagues [40] reported a highly significant correlation $(P=0.0004)$ between renal blood flow as estimated by CEUS and serum creatinine. In the same study, these investigators found that the determination of renal blood flow by CEUS reached a higher sensitivity (91 versus $82 \%$ ), specificity ( $82 \%$ versus $64 \%$ ) and accuracy (85 versus $73 \%$ ) for the diagnosis of chronic allograft nephropathy than conventional Doppler ultrasound. These findings where confirmed by Benozzi and colleagues [41], who performed CEUS in 39 kidney recipients at 5, 25 and 30 days after grafting. These researchers were able to show that some CEUS-derived parameters allowed the distinction between acute tubular necrosis and acute rejection episodes (cortico-medullar ratios of the rBV and mTT were lower in the acute tubular necrosis group compared to the control group, while another parameter, the time to peak, was higher than control in acute rejection events).

\section{Perspectives: CEUS in acute kidney injury}

CEUS is able to determine and quantify changes in renal perfusion and these changes play a role in clinical outcome as illustrated by the important findings in transplant medicine. In the critically ill patient, alterations in renal perfusion are expected to be found in various situations, such as septic shock, low cardiac output states, hepato-renal syndrome, and hypovolemia. However, it is still unclear whether these alterations are significant and related to renal outcome, whether they assist in diagnosis and early intervention or whether they represent the consequence rather than the cause of tubular injury. Given such uncertainty and the need to make rapid and repeated measurements early in the course of a patient's illness, CEUS could become an important tool to evaluate and quantify renal perfusion alterations in these different pathologic conditions. More importantly, CEUS might enable the study of severity, timing, and change over time of renal perfusion as well as the intra-renal distribution of perfusion abnormalities. In a second step, these observations might help draw a link between perfusion abnormalities as shown by CEUSderived parameters and AKI, and establish therapeutic targets and surrogate markers of adequate renal resuscitation. Finally, CEUS could be used to evaluate the renal perfusion effect of several of the hemodynamic interventions applied to patient care within the ICU.

However, in order to develop and validate this CEUS approach (as done in transplant medicine), specifically designed studies need to be performed in the setting of 
Table 2. CEUS-derived parameters in a 60-year-old patient before and after coronary artery bypass graft surgery complicated by acute kidney injury stage I

\begin{tabular}{|c|c|c|c|c|c|c|}
\hline & & \multicolumn{2}{|c|}{ Pre-op } & \multicolumn{2}{|c|}{ Post-op } & \multirow[b]{2}{*}{ Difference } \\
\hline & & Value & Average & Value & Average & \\
\hline \multirow[t]{3}{*}{ Mean transit time (mTT; s) } & AOI 1 & 1.51 & 1.91 & 2.06 & 2.09 & $+9.5 \%$ \\
\hline & $\mathrm{AOI} 2$ & 1.86 & & 2.03 & & \\
\hline & $\mathrm{AOI} 3$ & 2.35 & & 2.18 & & \\
\hline \multirow[t]{3}{*}{ Relative blood volume (rBV; a.u.) } & AOI 1 & 20,073 & 20,100 & 16,896 & 16,239 & $-19.2 \%$ \\
\hline & $\mathrm{AOI} 2$ & 19,309 & & 16,574 & & \\
\hline & $\mathrm{AOI} 3$ & 20,920 & & 15,248 & & \\
\hline \multirow[t]{2}{*}{ Perfusion index (= rBV/mTT; a.u.) } & AOI 1 & 13,293 & 10,523 & 8,202 & 7,786 & $-26.0 \%$ \\
\hline & $\mathrm{AOI} 3$ & 8,902 & & 6,994 & & \\
\hline
\end{tabular}

The mean transit time (or the 'speed' of replenishment) increased by close to $10 \%$ after surgery compared to baseline. In the same time, the relative blood volume (rBV; or echo density) decreased by almost $20 \%$. Altogether, the resulting perfusion index dropped by $26 \%$. This indicates diminished renal perfusion. rBV is a measure of pixel luminance and is proportional to local contrast agent concentration. This value does not have a physical unit (a.u. = arbitrary units) and is thus not comparable from one ultrasound machine to another, but its modifications are proportional to changes in contrast agent concentration. The software applies a linearization of the video data to generate a number proportional to the concentration of contrast. This operation aims to reverse the log compression applied by all ultrasound machines and is based on the pixel luminance. The luminance observed in each pixel is transformed by a program that attributes a value between 0 and 255 to each pixel. This value is then squared to reflect signal power. Possible values are between 0 and $255 \wedge 2$. These values do not have physical units but are proportional to the local concentration of contrast agent. In terms of absolute number, the values are not comparable between different systems (ultrasound machines) or different settings of a same system, but the kinetics of these values are respected. AOI, area of interest.

the ICU to investigate the various CEUS-derived parameters in the main syndromes known to be associated with AKI and to develop and validate CEUS-derived indices as it was done in transplant medicine. The characteristics of CEUS appear to make this approach uniquely possible.

\section{CEUS in the ICU}

CEUS is particularly well designed for use in the ICU. It combines the advantages of being fast, safe, non-invasive and repeatedly applicable at the bedside. Second generation ultrasound contrast agents are safe and well tolerated. This should allow repeated scans throughout the day to monitor the evolution of renal perfusion and response to treatment. The kidney is relatively easy to scan in the supine position and obtaining good quality images is relatively easy in most ICU patients. The technology allowing renal perfusion evaluation is still under improvement but our initial experience shows that CEUS can detect a $15 \%$ decrease in renal blood flow. This level of sensitivity is probably well above the value of changes expected to occur during the major hemodynamic events/syndrome known to be associated with AKI [4]. Thus, it seems unlikely that false negative examinations would occur with this technology.

\section{Conclusion}

CEUS is a safe, non-invasive and reliable technique. In many ways, it is ideally designed to monitor renal blood flow in ICU patients. Studies in renal transplant patients have shown its potential utility in clinical practice. Similar studies should now be performed in ICU patients to determine whether CEUS parameters predict or facilitate the early diagnosis of AKI and whether they can help assess the impact of therapeutic interventions in real time. CEUS would then logically help identify patients at risk of AKI at an earlier time and allow clinicians to adapt therapy to optimize renal perfusion and perhaps prevent AKI.

\section{Abbreviations}

AKI, acute kidney injury; CEUS, contrast-enhanced ultrasonography; MI, mechanical index; mTT, mean transit time; PI, perfusion index; rBV, relative blood volume.

\section{Competing interests}

The authors declare that they have no competing interests. Bracco SpA provided ultrasound contrast agent for the clinical studies at no cost.

\section{Acknowledgments}

The authors want to thank Michel Schneider, Marcel Arditi and Nicolas Rognin from Bracco Research Geneva SpA for their precious help and collaboration.

\section{Author details}

IIntensive Care Unit, Austin Hospital, 145 Studley Road, 3084 Heidelberg, Victoria, Australia. ${ }^{2}$ Australian and New Zealand Intensive Care Research Centre, School of Public Health and Preventive Medicine, Monash University, 3004 Melbourne, Victoria, Australia. ${ }^{3}$ Radiology department, Austin Hospital, 145 Studley Road, 3084 Heidelberg, Victoria, Australia.

Published: 6 May 2011

\section{References}

1. Uchino S, Kellum JA, Bellomo R, Doig GS, Morimatsu H, Morgera S, Schetz M, Tan I, Bouman C, Macedo E, Gibney N, Tolwani A, Ronco C; Beginning and 
Ending Supportive Therapy for the Kidney (BEST Kidney) Investigators: Acute renal failure in critically ill patients: a multinational, multicenter study. JAMA 2005, 294:813-818.

2. Levy EM, Viscoli CM, Horwitz RI: The effect of acute renal failure on mortality. A cohort analysis. JAMA 1996, 275:1489-1494.

3. Chertow GM, Levy EM, Hammermeister KE, Grover F, Daley J: Independent association between acute renal failure and mortality following cardiac surgery. Am J Med 1998, 104:343-348.

4. Prowle JR, Ishikawa K, May CN, Bellomo R: Renal blood flow during acute renal failure in man. Blood Purif 2009, 28:216-225

5. Selkurt EE: Renal blood flow and renal clearance in hemorraghic shock. Am J Physiol 1946, 145:699-799.

6. Wan L, Yang N, Hiew CY, Schelleman A, Johnson L, May C, Bellomo R: An assessment of the accuracy of renal blood flow estimation by Doppler ultrasound. Intensive Care Med 2008, 34:1503-1510.

7. Hofmann L, Simon-Zoula S, Nowak A, Giger A, Vock P, Boesch C, Frey FJ, Vogt $B$ : BOLD-MRI for the assessment of renal oxygenation in humans: acute effect of nephrotoxic xenobiotics. Kidney Int 2006, 70:144-150.

8. Prowle JR, Molan MP, Hornsey E, Bellomo R: Cine phase-contrast magnetic resonance imaging for the measurement of renal blood flow. Contrib Nephrol 2010, 165:329-336.

9. Geleskie JV, Shung KK: Further studies on acoustic impedance of major bovine blood vessel walls. J Acoust Soc Am 1982, 71:467-470.

10. Ophir J, Parker KJ: Contrast agents in diagnostic ultrasound. Ultrasound Med Biol 1989, 15:319-333.

11. Schacherer D, Klebl F, Goetz D, Buettner R, Zierhut S, Schoelmerich J, Langgartner J: Abdominal ultrasound in the intensive care unit: a 3-year survey on 400 patients. Intensive Care Med 2007, 33:841-844.

12. de Jong N, Ten Cate FJ, Lancee CT, Roelandt JR, Bom N: Principles and recent developments in ultrasound contrast agents. Ultrasonics 1991, 29:324-330.

13. Raisinghani A, Rafter P, Phillips P, Vannan MA, DeMaria AN: Microbubble contrast agents for echocardiography: rationale, composition, ultrasound interactions, and safety. Cardiol Clin 2004, 22:171-180, v.

14. Romero JR, Frey JL, Schwamm LH, Demaerschalk BM, Chaliki HP, Parikh G, Burke RF, Babikian VL: Cerebral ischemic events associated with 'bubble study' for identification of right to left shunts. Stroke 2009, 40:2343-2348

15. Feinstein SB, Cheirif J, Ten Cate FJ, Silverman PR, Heidenreich PA, Dick C, Desir RM, Armstrong WF, Quinones MA, Shah PM: Safety and efficacy of a new transpulmonary ultrasound contrast agent: initial multicenter clinical results. J Am Coll Cardio/ 1990, 16:316-324.

16. Klibanov A: Ultrasound contrast agents: development of the field and current status. Topics Curr Chem 2002, 222:73-106.

17. Morel DR, Schwieger I, Hohn L, Terrettaz J, Llull JB, Cornioley YA, Schneider M: Human pharmacokinetics and safety evaluation of SonoVue, a new contrast agent for ultrasound imaging. Invest Radio/ 2000, 35:80-85.

18. Cosgrove D: Angiogenesis imaging - ultrasound. Br J Radiol 2003, 76 Spec No 1:S43-49.

19. Schneider M: Bubbles and microcirculatory disorders. Eur Radio/ 2001, 11 Suppl 3:E1-5.

20. Mulvagh SL, Rakowski H, Vannan MA, Abdelmoneim SS, Becher H, Bierig SM, Burns PN, Castello R, Coon PD, Hagen ME, Jollis JG, Kimball TR, Kitzman DW, Kronzon I, Labovitz AJ, Lang RM, Mathew J, Moir WS, Nagueh SF, Pearlman AS, Perez JE, Porter TR, Rosenbloom J, Strachan GM, Thanigaraj S, Wei K, Woo A, Yu EH, Zoghbi WA; American Society of Echocardiography: American Society of Echocardiography Consensus Statement on the Clinical Applications of Ultrasonic Contrast Agents in Echocardiography. J Am Soc Echocardiogr 2008, 21:1179-1201; quiz 1281.

21. Fisher NG, Christiansen JP, Leong-Poi $H$, Jayaweera AR, Lindner JR, Kaul S: Myocardial and microcirculatory kinetics of BR14, a novel third-generation intravenous ultrasound contrast agent. J Am Coll Cardio/ 2002, 39:530-537.

22. Lindner JR, Song J, Jayaweera AR, Sklenar J, Kaul S: Microvascular rheology of Definity microbubbles after intra-arterial and intravenous administration. J Am Soc Echocardiogr 2002, 15:396-403.

23. Braide M, Rasmussen $\mathrm{H}$, Albrektsson A, Bagge U: Microvascular behavior and effects of sonazoid microbubbles in the cremaster muscle of rats after local administration. J Ultrasound Med 2006, 25:883-890.

24. Kusnetzky LL, Khalid A, Khumri TM, Moe TG, Jones PG, Main ML: Acute mortality in hospitalized patients undergoing echocardiography with and without an ultrasound contrast agent: results in 18,671 consecutive studies. J Am Coll Cardiol 2008, 51:1704-1706.
25. Dolan MS, Gala SS, Dodla S, Abdelmoneim SS, Xie F, Cloutier D, Bierig M, Mulvagh SL, Porter TR, Labovitz AJ: Safety and efficacy of commercially available ultrasound contrast agents for rest and stress echocardiography a multicenter experience. J Am Coll Cardiol 2009, 53:32-38.

26. Main ML, Ryan AC, Davis TE, Albano MP, Kusnetzky LL, Hibberd M: Acute mortality in hospitalized patients undergoing echocardiography with and without an ultrasound contrast agent (multicenter registry results in 4,300,966 consecutive patients). Am J Cardiol 2008, 102:1742-1746.

27. Wei K, Mulvagh SL, Carson L, Davidoff R, Gabriel R, Grimm RA, Wilson S, Fane L, Herzog CA, Zoghbi WA, Taylor R, Farrar M, Chaudhry FA, Porter TR, Irani W, Lang RM: The safety of deFinity and Optison for ultrasound image enhancement: a retrospective analysis of 78,383 administered contrast doses. J Am Soc Echocardiogr 2008, 21:1202-1206.

28. Jiménez C, de Gracia R, Aguilera A, Alonso S, Cirugeda A, Benito J, Regojo RM Aguilar R, Warlters A, Gómez R, Largo C, Selgas R: In situ kidney insonation with microbubble contrast agents does not cause renal tissue damage in a porcine model. J Ultrasound Med 2008, 27:1607-1615.

29. Wei K, Jayaweera AR, Firoozan S, Linka A, Skyba DM, Kaul S: Quantification of myocardial blood flow with ultrasound-induced destruction of microbubbles administered as a constant venous infusion. Circulation 1998, 97:473-483.

30. Wei K, Le E, Bin JP, Coggins M, Thorpe J, Kaul S: Quantification of renal blood flow with contrast-enhanced ultrasound. J Am Coll Cardiol 2001, 37:1135-1140

31. Tiemann K, Becher H, Lohmeier S: Real-time assessment of tissue perfusion following bubble destruction at low emission-power - first experimental results using power pulse inversion imaging. J Am Coll Cardiol 2000, 35:426A.

32. Arditi M, Frinking PJ, Zhou X, Rognin NG: A new formalism for the quantification of tissue perfusion by the destruction-replenishment method in contrast ultrasound imaging. IEEE Trans Ultrason Ferroelectr Frea Control 2006, 53:1118-1129.

33. Rim SJ, Leong-Poi H, Lindner JR, Couture D, Ellegala D, Mason H, Durieux M, Kassel NF, Kaul S: Quantification of cerebral perfusion with "real-time" contrast-enhanced ultrasound. Circulation 2001, 104:2582-2587.

34. Vogel R, Indermuhle A, Reinhardt J, Meier P, Siegrist PT, Namdar M, Kaufmann $P A$, Seiler $C$ : The quantification of absolute myocardial perfusion in humans by contrast echocardiography: algorithm and validation. J Am Coll Cardiol 2005, 45:754-762.

35. Kishimoto N, Mori Y, Nishiue T, Shibasaki Y, Iba O, Nose A, Uchiyama-Tanaka Y, Masaki H, Matsubara H, Iwasaka T: Renal blood flow measurement with contrast-enhanced harmonic ultrasonography: evaluation of dopamineinduced changes in renal cortical perfusion in humans. Clin Nephrol 2003, 59:423-428.

36. Kishimoto N, Mori Y, Nishiue T, Nose A, Kijima Y, Tokoro T, Yamahara H, Okigak M, Kosaki A, Iwasaka T: Ultrasound evaluation of valsartan therapy for rena cortical perfusion. Hypertens Res 2004, 27:345-349.

37. Kalantarinia K, Belcik JT, Patrie JT, Wei K: Real-time measurement of renal blood flow in healthy subjects using contrast-enhanced ultrasound. Am J Physiol Renal Physiol 2009, 297:F1129-1134.

38. Schneider A, Eggimann P, Burnier M, Vogt B: Renal perfusion quantification with contrast echography [abstract]. Swiss Med Forum 2009, 9(Suppl 48):7S

39. Fischer T, Dieckhofer J, Muhler M, Lembcke A, Morgera S, Budde K, Neumaye $H H$, Ebeling V, Thomas A, Filimonow S: The use of contrast-enhanced US in renal transplant: first results and potential clinical benefit. Eur Radiol 2005, 15 Suppl 5:E109-116.

40. Schwenger V, Korosoglou G, Hinkel UP, Morath C, Hansen A, Sommerer C, Dikow R, Hardt S, Schmidt J, Kücherer H, Katus HA, Zeier M: Real-time contrast-enhanced sonography of renal transplant recipients predicts chronic allograft nephropathy. Am J Transplant 2006, 6:609-615.

41. Benozzi L, Cappelli G, Granito M, Davoli D, Favali D, Montecchi MG, Grossi A, Torricelli P, Albertazzi A: Contrast-enhanced sonography in early kidney graft dysfunction. Transplant Proc 2009, 41:1214-1215.

\section{doi:10.1186/cc10058}

Cite this article as: Schneider $A$, et al:: Bench-to-bedside review: Contrast enhanced ultrasonography - a promising technique to assess renal perfusion in the ICU. Critical Care 2011, 15:157. 